\title{
Mathematical modeling of probe measurements in a supersonic flow of a four- component collisionless plasma
}

\author{
Institute of Technical Mechanics \\ of the National Academy of Sciences of U kraine and the State Space Agency of U kraine \\ 15 Leshko-Popel St., D nipro 49600, U kraine, e-mail: Iazuch.dn@ gmail.com
}

The aim of this work is the development of a procedure for extracting the plasma electron density and temperature and ion composition from the current-voltage characteristic ( $\mathrm{C}-\mathrm{V}$ characteristic) of an isolated probe system of cylindrical electrodes. The plasma is four-component and consists of electrons, ions of two species with significantly different masses, and neutrals. The measuring probe and the reference electrode of the probe system may be made up of several cylinders. The electrodes of the probe system are placed transversely to a supersonic flow of a low-temperature collisionless plasma with a specified mass velocity.

Using the familiar theoretical and experimental relationships for the ion and electron currents to a cylinder, a mathematical model of current collection is constructed for an isolated probe system at an arbitrary ratio of the electrode surface areas. The model includes the calculation of the equilibrium potential of the reference electrode as a function of the probe bias voltage. A procedure is developed for the identification of local plasma parameters using a priori information on the plasma properties and the experimental conditions. The effect of the electron density and temperature and the ion composition on the probe current of the isolated probe system at different ratios of the current-collecting electrode surface areas is studied. The ranges of the probe bias potentials and the values of the electrode surface area ratio that maximize and minimize the effect of the sought-for parameters on the probe current are determined. The quantitative restrictions on the bias potentials and the surface area ratio obtained in this study are used in the probe measurement procedure and in the objective function for comparing the theoretical approximation of the probe current with the measured I- characteristics.

A numerical simulation of probe measurements under the ionospheric conditions was conducted to verify the efficiency of the procedure for extracting the local parameters of a four-component plasma from the electron branch of the $\mathrm{I}-\mathrm{V}$ characteristic of an isolated probe system. The results obtained may be used in ionospheric plasma diagnostics onboard nanosatellites.

Keywords plasma ions of two species, isolated probe system with cylindrical electrodes, mathematical model of current collection, parametric identification, a priori information

1. Boyd R. L. F. Langmuir probes on spacecraft, in Plasma Diagnostics. Lochte-Holtgreven (Ed.). Amsterdam: North-Holland Publ. Co., 1968. Pp. 732-776.

2. Chung, P. M., Talbot L., Touryan K. J. Electric Probes in Stationary and Flowing Plasmas. SpringerVerlag, 1975. $150 \mathrm{pp}$ https://doi.org/10.1007/978-3-642-65886-0

3. Online resource - https://ccmc.gsfc.nasa.gov/modelweb/models/iri2012_vitmo.php.

4. Lazuchenkov D. N., Lazuchenkov N. M. Interpretation of probe measurements in a collisionless plasma flow. Teh. Meh. 2018. No. 1. Pp. 107-120. (in Russian). https://doi.org/10.15407/itm2018.01.107

5. Lazuchenkov D. N., Lazuchenkov N. M. Simulation of the extraction of the kinetic parameters of the ionospheric plasma from the current-voltage characteristics of a cylindrical probe. Teh. Meh. 2019. No. 4. Pp. 107-118. (in Russian). https://doi.org/10.15407/itm2019.04.107

6. Lazuchenkov D. N., Lazuchenkov N. M. Determination of the parameters of a supersonic dissociated rarefied plasma flow from the current-voltage characteristics of an isolated system of cylindrical probes. Teh. Meh. 2020. No. 2. Pp. 80-88. https://doi.org/10.15407/itm2020.02.080

7. Mott-Smith H., Langmuir I. The theory of collectors in gaseous discharges. Phys. Rev. 1926. V. 28. No. 5. Pp. 727-763. https://doi.org/10.1103/PhysRev.28.727

8. Hoegy W. R., Wharton L. E., Current to a moving cylindrical electrostatic probe. Journal of Applied Physics. 1973. V. 44. No. 12. Pp. 5365-5371. https://doi.org/10.1063/1.1662157 
9. Laframboise J. G. Theory of Spherical and Cylindrical Langmuir Probes in a Collisionless Maxwellian Plasma at Rest. Report No. 100. Univ. of Toronto, Institute of Aerospace Studies. 1966. 210 pp. https://doi.org/10.21236/AD0634596

10. Godard R., Laframboise J. Total current to cylindrical collectors in collisionless plasma flow. Space Science. 1983. V. 31, No. 3. p. 275-283.

https://doi.org/10.1016/0032-0633(83)90077-6

Received on November 5, 2020 in final form on November 24, 2020 